\title{
A Hand-Foot-and-Mouth Disease Model with Periodic Transmission Rate in Wenzhou, China
}

\author{
Yeting Zhu, ${ }^{1}$ Boyang Xu, ${ }^{1}$ Xinze Lian, ${ }^{1}$ Wang Lin, ${ }^{1}$ Zumu Zhou, ${ }^{2}$ and Weiming Wang ${ }^{1}$ \\ ${ }^{1}$ College of Mathematics and Information Science, Wenzhou University, Wenzhou 325035, China \\ ${ }^{2}$ Wenzhou Center for Disease Control and Prevention, Wenzhou 325000, China \\ Correspondence should be addressed to Weiming Wang; weimingwang2003@163.com
}

Received 11 January 2014; Revised 18 February 2014; Accepted 20 February 2014; Published 25 March 2014

Academic Editor: Kaifa Wang

Copyright (C) 2014 Yeting Zhu et al. This is an open access article distributed under the Creative Commons Attribution License, which permits unrestricted use, distribution, and reproduction in any medium, provided the original work is properly cited.

\begin{abstract}
We establish an SEIQRS epidemic model with periodic transmission rate to investigate the spread of seasonal HFMD in Wenzhou. The value of this study lies in two aspects. Mathematically, we show that the global dynamics of the HFMD model can be governed by its reproduction number $R_{0}$; if $R_{0}<1$, the disease-free equilibrium of the model is globally asymptotically stable, which means that the disease will vanish after some period of time; while if $R_{0}>1$, the model has at least one positive periodic solution and is uniformly persistent, which indicates that HFMD becomes an endemic disease. Epidemiologically, based on the statistical data of HFMD in Wenzhou, we find that the HFMD becomes an endemic disease and will break out in Wenzhou. One of the most interesting findings is that, for controlling the HFMD spread, we must increase the quarantined rate or decrease the treatment cycle.
\end{abstract}

\section{Introduction}

Hand, foot, and mouth disease (HFMD) was first reported in New Zealand in 1957 and is caused by Coxsackievirus A16 (CVA16) and human enterovirus 71 (HEV71) and occasionally by Coxsackie virus A4-A7, A9, A10, B1-B3, and B5. HFMD is an acute viral illness that usually affects infants and children younger than 5 years old, and, however, it can sometimes occur in adults. HFMD is characterized by fever, intraoral vesicles and erosions, and papulovesicles that favor the palms and soles. HFMD is spread from person to person through nose and throat secretions (such as saliva, sputum, or nasal mucus), blister fluid, or stool of infected persons [1, 2].

HFMD is moderately contagious and usually not a serious illness among the infected population; however, recent outbreak of HFMD in countries such as China, Singapore, and Finland has brought the world attention to HFMD due to complications of death related cases [3-10]. In 2008, there were 488,955 cases reported, with a morbidity of $37 / 100,000$, mortality of $0.0095 / 100,000$, and ill-death rate of $0.26 / 1000$; while, in 2009, there were 1,155,525 cases reported [11]. Due to the severity of this disease, the Ministry of Health of the People's Republic of China upgraded HFMD to a Class C communicable disease on May 2, 2008.

It is obvious that HFMD not only causes health problems but also has great social and economic impacts which are not easily quantifiable. So it is important to understand the spread dynamics of HFMD among the susceptible populations and to enable policy makers to take effective measures to curb the disease spread and reduce the adverse impact of the disease $[12,13]$.

There are several types of analytical models that are valuable to understand and predict transmission of HFMD. One is the statistical models which can help us find novel information concerning pathogen detection and some probable coinfection factors in HFMD and have been applied to understand HFMD's spatiotemporal transmission and discover the relationship between HFMD occurrence and climate $[2-11,14,15]$. The other is compartmental differential equation model $[12,13,16-20]$. Of them, Tiing and Labadin [16] were the first to propose a simple SIR epidemic model to predict the number of the infected and the duration of an outbreak when HFMD occurs and found that the disease spread quite rapidly and the parameter that may be able to 
be controlled would be the number of susceptible persons. Roy and Haider [12] and Roy [13] established a simple SEIR model to understand the dynamics of HFMD among young children and found that disease transmission depended more on the number of actively infected people in the population at the initial time and could be controlled by quarantine of more actively infected individuals. Wang and Xue [18] and Yang et al. [19] established SEIQR (susceptible-exposedinfectious-quarantined-recovered) model, respectively, and estimated the basic reproduction number for the HFMD transmission in Mainland China and predicted that HFMD was an endemic disease in China.

After a susceptible individual is infected, he/she firstly enter the incubation period of HFMD, which period is about 3 to 7 days. After the incubation period, the infected will show some clinical symptoms, such as having a fever, poor appetite, malaise, and sore throat, and few people may develop dehydration, febrile seizures, encephalitis, meningitis, cardiomyopathy, and so forth. And the infected people will fully recover after 7 to 10 days [1]. The occurrence of HFMD has seasonal characteristics and is often associated with climate changes $[11,21]$. Periodic changing in the birth rate [22] and seasonally changing in the contact rate [23] are often regarded as sources of periodicity.

In the sense of seasonally changing in the contact rate, Liu [17] and Ma et al. [20] established HFMD with periodic transmission rate, respectively. In [17], Liu constructed a periodic SEIQR epidemic model to simulate the dynamics of HFMD transmission and showed that quarantine in the children population had a positive impact on controlling the spread of HFMD. And Ma et al. [20] proposed a SEIIeQR HFMD model, and found that the recessive subpopulation played an important role in the spread of HFMD, and only quarantining the infected is not an effective measure in controlling the disease.

Wenzhou is a prefecture-level city in southeastern Zhejiang, province in China. At the time of the 2010 Chinese census, 9,122,100 people lived in Wenzhou [24]. Since Wenzhou has a humid subtropical climate zone with an annual average $18.08^{\circ} \mathrm{C}\left(64.5^{\circ} \mathrm{F}\right)$, it is of particular public health significance to update molecular epidemiology of HFMD in Wenzhou. It is reported that, in 2012, there were 147,941 HFMD cases and 17 deaths in Zhejiang province, and it is the first of the "ten legal infectious diseases"; there were 41,438 HFMD cases and 4 deaths in Wenzhou [25]. The numbers of weekly reported HFMD cases in Wenzhou from March 5, 2010, to December 27, 2013, are listed in Table 1.

The aim of this study is to use mathematical modeling to gain some insights into the transmission dynamics of HFMD in Wenzhou and to assess the aforementioned preventive strategies. In particular, we aim to answer the following questions through our analytic and numerical results of the HFMD model.

(1) What is the disease dynamics of the HFMD in Wenzhou?

(2) How can we control the HFMD spread in Wenzhou?

The paper is organized as follows. In Section 2, we derive a SEIQRS HFMD model. In Section 3, we give the main theoretical results of the HFMD model. In Section 4, based on the HFMD data of the Wenzhou Center for Disease Control and Prevention, we perform some simulation results of the model and sensitivity analysis. In Section 5, we give a brief discussion.

\section{Model Derivation}

In order to establish an HFMD model in Wenzhou, we classify the population into five compartments according to their states: susceptible, exposed, infected, quarantined, and recovered, which are denoted by $S(t), E(t), I(t), Q(t)$, and $R(t)$, respectively. We denote the total population by $N(t)$, that is $N(t)=S(t)+E(t)+I(t)+Q(t)+R(t)$. And the nonautonomous differential equations for HFMD model are

$$
\begin{gathered}
\frac{d S}{d t}=\Lambda-d S-\frac{\beta(t) S I}{S+\alpha I}+\eta R, \\
\frac{d E}{d t}=\frac{\beta(t) S I}{S+\alpha I}-(d+\sigma) E, \\
\frac{d I}{d t}=\sigma E-\left(d+\delta_{1}+k+\gamma_{1}\right) I, \\
\frac{d Q}{d t}=k I-\left(d+\delta_{2}+\gamma_{2}\right) Q, \\
\frac{d R}{d t}=\gamma_{1} I+\gamma_{2} Q-(d+\eta) R,
\end{gathered}
$$

and the meaning and units of each variable and constant in model (1) are as follows:

$\Lambda$ : the recruitment rate of susceptible $\left(\right.$ week $^{-1}$ ), $d$ : the per capita natural mortality rate $\left(\right.$ week $\left.^{-1}\right)$,

$\beta(t)$ : the periodic transmission rate coefficient, which is a continuous, positive $\omega$-periodic function,

$\alpha(0<\alpha \leq 1)$ : the "psychological" effect, which can be interpreted as follows: when an infectious disease appears and spreads in a region, people deter from risky behavior or from taking precautionary measures in relation to the disease outbreak; human behavior change consequently leads to reduction in number of real susceptible individuals or effective contact rates [26],

$1 / \sigma$ : the mean incubation period (week),

$k$ : the quarantined rate $\left(\right.$ week $\left.^{-1}\right)$; this means that some of the infected people will be hospitalized for treatment, and, thus, these quarantined $Q(t)$ are isolated from other subpopulations,

$\gamma_{1}$ : the infectious recover rate, which means that the infectious individuals recover and return to recovered compartment $R$ from compartments $I$ (week),

$\gamma_{2}$ : the quarantined rate, which means that the quarantined individuals recover and return to recovered compartment $R$ from compartments $Q$ (week),

$\delta_{1}$ : the disease-induced mortality for the infective individuals ( week $^{-1}$ ), 
TABLE 1: The numbers of weekly reported HFMD cases in Wenzhou.

\begin{tabular}{|c|c|c|c|c|c|c|c|c|c|c|c|c|c|c|c|}
\hline Week & Data & Week & Data & Week & Data & Week & Data & Week & Data & Week & Data & Week & Data & Week & Data \\
\hline 1 & 368 & 26 & 331 & 51 & 46 & 76 & 366 & 101 & 164 & 126 & 469 & 151 & 238 & 176 & 749 \\
\hline 2 & 380 & 27 & 320 & 52 & 47 & 77 & 371 & 102 & 193 & 127 & 413 & 152 & 189 & 177 & 794 \\
\hline 3 & 457 & 28 & 475 & 53 & 136 & 78 & 317 & 103 & 234 & 128 & 413 & 153 & 145 & 178 & 708 \\
\hline 4 & 615 & 29 & 415 & 54 & 153 & 79 & 291 & 104 & 226 & 129 & 467 & 154 & 141 & 179 & 600 \\
\hline 5 & 581 & 30 & 339 & 55 & 197 & 80 & 441 & 105 & 264 & 130 & 429 & 155 & 131 & 180 & 518 \\
\hline 6 & 694 & 31 & 352 & 56 & 366 & 81 & 549 & 106 & 456 & 131 & 439 & 156 & 118 & 181 & 505 \\
\hline 7 & 924 & 32 & 201 & 57 & 322 & 82 & 395 & 107 & 478 & 132 & 676 & 157 & 223 & 182 & 523 \\
\hline 8 & 1124 & 33 & 254 & 58 & 401 & 83 & 478 & 108 & 743 & 133 & 861 & 158 & 268 & 183 & 520 \\
\hline 9 & 1200 & 34 & 226 & 59 & 460 & 84 & 410 & 109 & 876 & 134 & 793 & 159 & 351 & 184 & 446 \\
\hline 10 & 1539 & 35 & 243 & 60 & 549 & 85 & 565 & 110 & 1035 & 135 & 765 & 160 & 455 & 185 & 510 \\
\hline 11 & 1744 & 36 & 182 & 61 & 574 & 86 & 508 & 111 & 1403 & 136 & 645 & 161 & 517 & 186 & 714 \\
\hline 12 & 1706 & 37 & 173 & 62 & 767 & 87 & 543 & 112 & 1660 & 137 & 491 & 162 & 596 & 187 & 917 \\
\hline 13 & 1528 & 38 & 187 & 63 & 1165 & 88 & 538 & 113 & 1908 & 138 & 542 & 163 & 577 & 188 & 672 \\
\hline 14 & 1329 & 39 & 214 & 64 & 1247 & 89 & 787 & 114 & 2240 & 139 & 600 & 164 & 517 & 189 & 589 \\
\hline 15 & 1252 & 40 & 186 & 65 & 1168 & 90 & 689 & 115 & 1974 & 140 & 644 & 165 & 552 & 190 & 482 \\
\hline 16 & 1447 & 41 & 166 & 66 & 1060 & 91 & 854 & 116 & 2163 & 141 & 579 & 166 & 546 & 191 & 369 \\
\hline 17 & 1663 & 42 & 192 & 67 & 1388 & 92 & 785 & 117 & 1545 & 142 & 691 & 167 & 668 & 192 & 326 \\
\hline 18 & 1346 & 43 & 190 & 68 & 1686 & 93 & 618 & 118 & 1611 & 143 & 777 & 168 & 874 & 193 & 366 \\
\hline 19 & 1247 & 44 & 181 & 69 & 1532 & 94 & 476 & 119 & 1868 & 144 & 783 & 169 & 1068 & 194 & 491 \\
\hline 20 & 1094 & 45 & 171 & 70 & 1323 & 95 & 414 & 120 & 1683 & 145 & 732 & 170 & 1266 & 195 & 402 \\
\hline 21 & 794 & 46 & 95 & 71 & 819 & 96 & 365 & 121 & 1631 & 146 & 561 & 171 & 1001 & 196 & 439 \\
\hline 22 & 673 & 47 & 66 & 72 & 743 & 97 & 308 & 122 & 1228 & 147 & 636 & 172 & 1037 & 197 & 371 \\
\hline 23 & 584 & 48 & 55 & 73 & 636 & 98 & 206 & 123 & 758 & 148 & 441 & 173 & 1363 & 198 & 358 \\
\hline 24 & 448 & 49 & 37 & 74 & 602 & 99 & 162 & 124 & 502 & 149 & 345 & 174 & 1051 & 199 & 382 \\
\hline 25 & 420 & 50 & 42 & 75 & 478 & 100 & 161 & 125 & 519 & 150 & 225 & 175 & 995 & & \\
\hline
\end{tabular}

$\delta_{2}$ : the disease-induced mortality for the quarantined individuals ( week $^{-1}$ ),

$\eta$ : the progression rate of the recovered individual $R$.

\section{Dynamics Analysis of the HFMD Model}

3.1. Preliminaries. Let $\left(\mathbb{R}^{n}, \mathbb{R}_{+}^{n}\right)$ be the standard ordered $n$ dimensional Euclidean space with a norm $\|\cdot\|$. For $u, v \in \mathbb{R}^{n}$, we denote $u \geq v$, if $u-v \in \mathbb{R}_{+}^{n} ; u>v$, if $u-v \in \mathbb{R}_{+}^{n} 0$; and $u \gg v$, if $u-v \in \operatorname{Int}\left(\mathbb{R}_{+}^{n}\right)$.

We briefly present some main results of Floquet theory. Consider the following linear periodic system:

$$
\frac{d \mathbf{u}}{d t}=A(t) \mathbf{u},
$$

where $\mathbf{u}$ is an $n \times 1$ vector and $A(t)$ is an $n \times n$ matrix of principal periodic $\omega>0$, such that $A(t+\omega)=A(t)$.

Let $\Phi_{A(\cdot)}(t)$ be a fundamental solution matrix of (2); that is, a nonsingular matrix each of the columns of which is a solution of equation such that $\Phi_{A(\cdot)}(0)=\mathbb{\square}$, the identify matrix. Floquet theory show that the fundamental matrix has the form $\Phi_{A(\cdot)}(t)=P(t) e^{B t}$, where $P(t)=P(t+\omega)$ is a periodic matrix with initial values $P(0)=\llbracket$ and $B$ is a constant matrix. The matrix $\Phi_{A(\cdot)}(T)=e^{B T}$ is the so-called principle or monodromy or Floquet transition matrix of (2). Let $r\left(\Phi_{A(\cdot)}(\omega)\right)$ be the spectral radius of $\Phi_{A(\cdot)}(\omega)$. It then follows from $[27,28]$ that $\Phi_{A(\cdot)}(\omega)$ is a matrix with all entries positive for each $t>0$. By the Perron-Frobenius theorem [29], $r\left(\Phi_{A(\cdot)}(\omega)\right)$ is the principal eigenvalue of $\Phi_{A(\cdot)}(\omega)$ in the sense that it is simple and admits an eigenvector $u^{*} \gg 0$. The following result is useful for our subsequent comparison arguments.

Lemma 1 (see [30, Lemma 2.1]). Let $p=(1 / \omega) \ln r\left(\Phi_{A(\cdot)}(\omega)\right)$. Then there exists a positive, $\omega$-periodic function $v(t)$ such that $e^{p t} v(t)$ is a solution of (2).

3.2. Positively Invariant Sets. The study of the dynamics of model (1) requires the introduction of the following important sets:

$$
\begin{gathered}
X=\left\{(S, E, I, Q, R) \in \mathbb{R}_{+}^{5}\right\}, \\
\Gamma=\left\{(S, E, I, Q, R) \in X, 0<S+E+I+Q+R \leq \frac{\Lambda}{d}\right\} .
\end{gathered}
$$

And we can easily obtain the following theorem of the positively invariant sets. 
Theorem 2 (positively invariant sets). $X$ and $\Gamma$ are positive invariant sets for model (1).

Proof. It shows that $X$ is positively invariant. From model (1), the total population $N(t)$ satisfies the following equation:

$$
\begin{aligned}
\Lambda-\left(d+\delta_{1}+\delta_{2}\right) N & \leq \frac{d N}{d t} \\
& =\Lambda-d N-\delta_{1} I-\delta_{2} Q \leq \Lambda-d N .
\end{aligned}
$$

Hence, by integration, we check

$$
\begin{gathered}
\frac{\Lambda}{d+\delta_{1}+\delta_{2}}+\left(N(0)-\frac{\Lambda}{d+\delta_{1}+\delta_{2}}\right) e^{-\left(d+\delta_{1}+\delta_{2}\right) t} \\
\leq N(t) \leq \frac{\Lambda}{d}+\left(N(0)-\frac{\Lambda}{d}\right) e^{-d t}
\end{gathered}
$$

Hence,

$$
\frac{\Lambda}{d+\delta_{1}+\delta_{2}} \leq \lim _{t \rightarrow \infty} \inf N(t) \leq \lim _{t \rightarrow \infty} \sup N(t) \leq \frac{\Lambda}{d},
$$

which implies that $\Gamma$ is positively invariant with respect to model (1).

3.3. The Basic Reproduction Number $R_{0}$. It is easy to see that model (1) always has one disease-free equilibrium $P_{0}=$ $(\Lambda / d, 0,0,0,0)$. By the definition of Bacaër and Guernaoui [31] and the general calculation procedure in Wang and Zhao [32], we have

$$
\mathscr{F}=\left(\begin{array}{c}
\frac{\beta(t) S I}{S+\alpha I} \\
0 \\
0 \\
0 \\
0
\end{array}\right),
$$

$$
\mathscr{V}^{-}=\left(\begin{array}{c}
0 \\
\sigma E \\
k I \\
\Lambda+\eta R \\
\gamma_{1} I+\gamma_{2} Q
\end{array}\right) \text {. }
$$

It follows that

$$
\begin{gathered}
F(t)=\left(\begin{array}{ccc}
0 & \beta(t) & 0 \\
0 & 0 & 0 \\
0 & 0 & 0
\end{array}\right), \quad M=\left(\begin{array}{cc}
-d & \eta \\
0 & -(d+\eta)
\end{array}\right), \\
V(t)=\left(\begin{array}{ccc}
d+\sigma & 0 & 0 \\
-\sigma & d+\delta_{1}+k+\gamma_{1} & 0 \\
0 & -k & d+\delta_{2}+\gamma_{2}
\end{array}\right) .
\end{gathered}
$$

Furthermore, $F(t)$ is nonnegative, and $-V(t)$ is cooperative in the sense that the off-diagonal elements of $-V(t)$ are nonnegative.

It is obvious that $-d$ and $-(d+\eta)$ are the eigenvalues of $M$ and are negative. Thus $M$ is stable; namely, $r\left(\phi_{M}(\omega)\right)<1$. We can see that the eigenvalues of $-V$ are the diagonal elements and are negative. So $-V$ is stable; namely, $r\left(\phi_{-V}(\omega)\right)<1$.

Let $Y(t, s), t \geq s$, be the evolution operator of the linear $\omega$-periodic system. Consider

$$
\frac{d y}{d t}=-V(t) y
$$

That is, for each $s \in \mathbb{R}$, the $3 \times 3$ matrix $Y(t, s)$ satisfies

$$
\frac{d Y(t, s)}{d t}=-V(t) Y(t, s), \quad \forall t \geq s, Y(s, s)=\mathbb{\square}
$$

where $\llbracket$ is the $3 \times 3$ identity matrix. Thus, the monodromy matrix $\Phi_{-V}(t)$ of (9) equals $Y(t, 0), t \geq 0$.

Following the method in Wang and Zhao [32], we let $\phi(s)$ be $\omega$-periodic in $s$ and the initial distribution of infectious individuals. So $F(s) \phi(s)$ is the rate of new infections produced by the infected individuals who are introduced at time $s$. When $t \geq s, Y(t, s) F(s) \phi(s)$ gives the distribution of those infected individuals who are newly infected by $\phi(s)$ and remain in the infected compartments at time $t$. Naturally,

$$
\begin{array}{rl}
\int_{-\infty}^{t} & Y(t, s) F(s) \phi(s) d s \\
& =\int_{0}^{\infty} Y(t, t-a) F(t-a) \phi(t-a) d a
\end{array}
$$

is the distribution of accumulative new infections at time $t$ produced by all those infected individuals $\phi(s)$ introduced at time previous to $t$.

Let $C_{\omega}$ be the ordered Banach space of all $\omega$-periodic functions from $\mathbb{R}$ to $\mathbb{R}^{3}$, which is equipped with the maximum norm $\|\cdot\|$ and the positive cone $C_{\omega}^{+}:=\left\{\phi \in C_{\omega}: \phi(t) \geq\right.$ $0, \forall t \in \mathbb{R}\}$. Then we can define a linear operator. Consider

$$
\begin{array}{r}
(L \phi)(t)=\int_{0}^{\infty} Y(t, t-a) F(t-a) \phi(t-a) d a, \\
\forall t \in \mathbb{R}, \phi \in C_{\omega} .
\end{array}
$$


$L$ is called the next infection operator and the spectral radius of $L$ is defined as the basic reproduction number. Consider

$$
R_{0}:=r(L) \text {, }
$$

for the periodic epidemic model.

Lemma 3 (see [32]). The following statements are valid:

(i) $R_{0}=1$ if and only if $r\left(\Phi_{F-V}(\omega)\right)=1$;

(ii) $R_{0}>1$ if and only if $r\left(\Phi_{F-V}(\omega)\right)>1$;

(iii) $R_{0}<1$ if and only if $r\left(\Phi_{F-V}(\omega)\right)<1$.

Thus, the disease-free equilibrium $P_{0}=(\Lambda / d, 0,0,0,0)$ of model (1) is asymptotically stable if $R_{0}<1$ and unstable if $R_{0}>1$.

Now we introduce the linear $\omega$-periodic system

$$
\frac{d w}{d t}=\left(-V(t)+\frac{F(t)}{\lambda}\right) w, \quad t \in \mathbb{R}_{+}
$$

with parameter $\lambda \in \mathbb{R}$. Let $W(t, s, \lambda), t \geq s$, be the evolution operator of system (14) on $\mathbb{R}^{4}$. Clearly, $\Phi_{F-V}(t)=$ $W(t, 0,1), t \geq 0$. Moreover,

$$
\begin{aligned}
& -V(t)+\frac{F(t)}{\lambda} \\
& =\left(\begin{array}{ccc}
-(d+\sigma) & \frac{\beta(t)}{\lambda} & 0 \\
\sigma & -\left(d+\delta_{1}+k+\gamma_{1}\right) & 0 \\
0 & k & -\left(d+\delta_{2}+\gamma_{2}\right)
\end{array}\right) .
\end{aligned}
$$

Hence, we derive

$$
\Phi_{F / \lambda-V}(t)=W(t, 0, \lambda)
$$

Following the general calculation procedure in Wang and Zhao [32], the basic reproduction number $R_{0}$ is the unique solution of $r(W(\omega, 0, \lambda))=1$.

\subsection{Extinction of the Disease}

Theorem 4. The disease-free equilibrium $P_{0}=(\Lambda / d, 0,0,0,0)$ of model (1) is globally asymptotically stable if $R_{0}<1$ and unstable if $R_{0}>1$.

Proof. If $R_{0}<1$, according to Lemma 3, we have $r\left(\Phi_{F-V}\right.$ $(\omega))<1$. From model (1), for $t \geq 0$, we know that

$$
\begin{gathered}
\frac{d E}{d t} \leq \beta(t) I-(d+\sigma) E, \\
\frac{d I}{d t}=\sigma E-\left(d+\delta_{1}+k+\gamma_{1}\right) I, \\
\frac{d Q}{d t}=k I-\left(d+\delta_{2}+\gamma_{2}\right) Q .
\end{gathered}
$$

Consider the following comparison system:

$$
\begin{gathered}
\frac{d \widetilde{E}}{d t}=\beta(t) \widetilde{I}-(d+\sigma) \widetilde{E}, \\
\frac{d \widetilde{I}}{d t}=\sigma \widetilde{E}-\left(d+\delta_{1}+k+\gamma_{1}\right) \widetilde{I}, \\
\frac{d \widetilde{Q}}{d t}=k \widetilde{I}-\left(d+\delta_{2}+\gamma_{2}\right) \widetilde{Q} ;
\end{gathered}
$$

that is,

$$
\frac{d g}{d t}=(F(t)-V(t)) g(t), \quad g(t)=(\widetilde{E}(t), \widetilde{I}(t), \widetilde{Q}(t))^{T} .
$$

By Lemma 1, there exists a positive $\omega$-periodic function $\widetilde{g}(t)$ such that $g(t)=e^{p t} \tilde{g}(t)$ is a solution of (18), where $p=$ $(1 / \omega) \ln r\left(\Phi_{F-V}(\omega)\right)<0$. Then we conclude that $g(t) \rightarrow 0$ as $t \rightarrow \infty$, which implies that the zero solution of model (18) is globally asymptotically stable. Applying the comparison principle, we known that $E(t) \rightarrow 0, I(t) \rightarrow 0$, and $Q(t) \rightarrow$ 0 as $t \rightarrow \infty$. It follows that $R(t) \rightarrow 0$, and $S(t) \rightarrow$ $\Lambda / d$ as $t \rightarrow \infty$. Therefore, the disease-free equilibrium $P_{0}=(\Lambda / d, 0,0,0,0)$ is globally asymptotically stable. This completes the proof.

Remark 5. From Theorem 4, one can know that if $R_{0}<1$, then $P_{0}=(\Lambda / d, 0,0,0,0)$ is globally asymptotically stable, which means that the disease will vanish after some period of time. Therefore, it is interesting to study the disease-free equilibrium for controlling infectious disease.

\subsection{Uniform Persistence of the Disease. Define}

$$
\Gamma_{0}:=\{(S, E, I, Q, R) \in \Gamma: E>0, I>0\}
$$

and $\partial \Gamma_{0}=\Gamma \backslash \Gamma_{0}=\{(S, E, I, Q, R) \in \Gamma: E(t) \equiv 0$ or $I(t) \equiv 0\}$. Denote $u\left(t, x_{0}\right)$ as the unique solution of model (1) with the initial value $x_{0}:=\left(S_{0}, E_{0}, I_{0}, Q_{0}, R_{0}\right)$. Let $P: \Gamma \rightarrow \Gamma$ be the Poincaré map associated with model (1); that is,

$$
P\left(x_{0}\right)=u\left(\omega, x_{0}\right), \quad \forall x_{0} \in \Gamma,
$$

where $\omega$ is the period. By applying the fundamental existenceuniqueness theorem [33], we know that $u\left(t, x_{0}\right)$ is the unique solution of model (1) with $u\left(0, x_{0}\right)=x_{0}$. From Theorem 2, we know that $P$ is dissipative point. We then introduce the following lemma.

Lemma 6. If $R_{0}>1$, then there exists a $\epsilon>0$ such that when $\left\|x_{0}-P_{0}\right\| \leq \epsilon$, for any $x_{0} \in \Gamma_{0}$, one has

$$
\limsup _{m \rightarrow \infty} d\left[P^{m}\left(x_{0}\right)-P_{0}\right] \geq \epsilon .
$$

Proof. We proceed by contradiction to prove that

$$
\limsup _{m \rightarrow \infty} d\left[P^{m}\left(x_{0}\right)-P_{0}\right] \geq \epsilon .
$$


If not, then

$$
\limsup _{m \rightarrow \infty} d\left[P^{m}\left(x_{0}\right)-P_{0}\right]<\epsilon,
$$

for some $x_{0} \in \Gamma_{0}$. Without loss of generality, we can assume that $d\left(P^{m}\left(x_{0}\right), P_{0}\right)<\epsilon$, for all $m \geq 0$. By the continuity of the solutions with respect to the initial values, for all $\varepsilon \in(0, \Lambda / d)$, we obtain

$$
\left\|u\left(t, P^{m}\left(x_{0}\right)\right)-u\left(t, P_{0}\right)\right\| \leq \varepsilon, \quad \forall t \in[0, \omega] .
$$

For any $t \geq 0$, let $t=m \omega+t_{1}$, where $t_{1} \in[0, \omega]$ and $m=[t / \omega]$, which is the greatest integer less than or equal to $t / \omega$. Then we have

$$
\begin{aligned}
\left\|u\left(t, P^{m}\left(x_{0}\right)\right)-u\left(t, P_{0}\right)\right\| & =\left\|u\left(t_{1}, P^{m}\left(x_{0}\right)\right)-u\left(t_{1}, P_{0}\right)\right\| \\
& \leq \varepsilon, \quad \forall t \geq 0 .
\end{aligned}
$$

It follows that $\Lambda / d-\varepsilon \leq S(t) \leq \Lambda / d+\varepsilon, 0 \leq I(t) \leq \varepsilon$, for $t \geq 0$. Then $S /(S+\alpha I) \geq 1-\alpha \varepsilon /(\Lambda / d+(\alpha-1) \varepsilon):=1-\theta>0$. Thus, for $\left\|x_{0}-P_{0}\right\| \leq \epsilon$, we have

$$
\begin{gathered}
\frac{d E}{d t} \geq \beta(t)(1-\theta) I-(d+\sigma) E \\
\frac{d I}{d t}=\sigma E-\left(d+\delta_{1}+k+\gamma_{1}\right) I \\
\frac{d Q}{d t}=k I-\left(d+\delta_{2}+\gamma_{2}\right) Q .
\end{gathered}
$$

We consider the following comparison system

$$
\begin{gathered}
\frac{d \bar{E}}{d t}=\beta(t)(1-\theta) \bar{I}-(d+\sigma) \bar{E} \\
\frac{d \bar{I}}{d t}=\sigma \bar{E}-\left(d+\delta_{1}+k+\gamma_{1}\right) \bar{I} \\
\frac{d \bar{Q}}{d t}=k \bar{I}-\left(d+\delta_{2}+\gamma_{2}\right) \bar{Q} .
\end{gathered}
$$

Set $M_{\varepsilon}=\left(\begin{array}{lll}0 & \theta & 0 \\ 0 & 0 & 0 \\ 0 & 0 & 0\end{array}\right)$. Then, model (28) can be rewritten as

$$
\begin{gathered}
\frac{d g}{d t}=\left(F(t)-V(t)-M_{\varepsilon}\right) g(t), \\
g(t)=(\bar{E}(t), \bar{I}(t), \bar{Q}(t))^{T} .
\end{gathered}
$$

If $R_{0}>1$, we obtain $r\left(\Phi_{F-V}(\omega)\right)>1$ by Lemma 3. Then we can choose $\varepsilon>0$ small enough such that $r\left(\Phi_{F-V-M_{\varepsilon}}(\omega)\right)>1$. Once again by Lemma 1, it follows that there exists a positive $\omega$-periodic $\bar{g}(t)$ such that $g(t)=e^{p_{1} t} \bar{g}(t)$ is a solution of model (28), where $p_{1}=(1 / \omega) \ln r\left(\Phi_{F-V}(\omega)\right)>0$, which implies that $g(t) \rightarrow \infty$ as $t \rightarrow \infty$. By applying the comparison principle, we have $E(t) \rightarrow \infty, I(t) \rightarrow \infty$, and before $Q(t) \rightarrow \infty$ as $t \rightarrow \infty$. This is a contraction.

By using Lemma 6, we can obtain the following theorem.
Theorem 7. If $R_{0}>1$, model (1) has at least one positive periodic solution and is uniformly persistent.

Proof. We first prove that $\left\{P^{m}\right\}_{m \geq 0}$ is uniformly persistent with respect to $\left(\Gamma_{0}, \partial \Gamma_{0}\right)$. First of all, we show that $\Gamma_{0}$ and $\partial \Gamma_{0}$ are positively invariant, for any $\left(S_{0}, E_{0}, I_{0}, Q_{0}, R_{0}\right) \in \Gamma_{0}$. From the first equation of model (1), we derive that

$$
\frac{d S}{d t} \geq \Lambda-\left(d+\frac{\beta(t)}{\alpha}\right) S+\eta R .
$$

So, we have

$$
\begin{aligned}
S(t) \geq & e^{-\int_{0}^{t}\left(d+\beta(s) \alpha^{-1}\right) d s} \\
& \times\left(S_{0}+\int_{0}^{t} e^{\int_{0}^{\tau}\left(d+\beta(s) \alpha^{-1}\right) d s}(\Lambda+\eta R(\tau)) d \tau\right)
\end{aligned}
$$

$$
>0, \quad \forall t>0 \text {. }
$$

Similarly,

$$
\begin{aligned}
E(t) & =e^{-(d+\sigma) t}\left(E_{0}+\int_{0}^{t} \frac{\beta(\tau) S(\tau) I(\tau)}{S(\tau)+\alpha I(\tau)} e^{(d+\sigma) \tau} d \tau\right) \\
& >0, \quad \forall t>0, \\
I(t) & =e^{-\left(d+\delta_{1}+k+\gamma_{1}\right) t}\left(I_{0}+\int_{0}^{t} \sigma E(\tau) e^{\left(d+\delta_{1}+k+\gamma_{1}\right) \tau} d \tau\right) \\
& >0, \quad \forall t>0, \\
Q(t) & =e^{-\left(d+\delta_{1}+k+\gamma_{1}\right) t}\left(Q_{0}+\int_{0}^{t} k I(\tau) e^{\left(d+\delta_{1}+k+\gamma_{1}\right) \tau} d \tau\right) \\
& >0, \quad \forall t>0, \quad \\
R(t) & =e^{-(d+\eta) t}\left(R_{0}+\int_{0}^{t}\left(\gamma_{1} I(\tau)+\gamma_{2} Q(\tau)\right) e^{(d+\eta) \tau} d \tau\right) \\
& >0, \quad \forall t>0 .
\end{aligned}
$$

Thus, $\Gamma_{0}$ is positively invariant. Clearly, $\partial \Gamma_{0}$ is relatively closed in $\Gamma$. Set

$$
\begin{gathered}
M_{\partial}=\left\{\left(S_{0}, E_{0}, I_{0}, Q_{0}, R_{0}\right) \in \partial \Gamma_{0}: P^{m}\left(S_{0}, E_{0}, I_{0}, Q_{0}, R_{0}\right)\right. \\
\left.\in \partial \Gamma_{0}, \forall m \geq 0\right\} .
\end{gathered}
$$

We claim that

$$
M_{\partial}=\{(S, 0,0, Q, R): S \geq 0, Q \geq 0, R \geq 0\} .
$$

Note that

$$
\{(S, 0,0, Q, R): S \geq 0, Q \geq 0, R \geq 0\} \subseteq M_{\partial} .
$$

We only need to prove that

$$
M_{\partial} \subseteq\{(S, 0,0, Q, R): S \geq 0, Q \geq 0, R \geq 0\} .
$$

In fact, for any $\left(S_{0}, E_{0}, I_{0}, Q_{0}, R_{0}\right) \in \partial \Gamma_{0} \backslash\{(S, 0,0, Q, R): S \geq$ $0, Q \geq 0, R \geq 0\}$, in the case where $E_{0}=0, I_{0}>0$, it is clear 
that $S(t)>0, I(t)>0$, for all $t>0$. From the second equation of model (1), we have

$$
\begin{aligned}
E(t) & =e^{-(d+\sigma) t}\left(E_{0}+\int_{0}^{t} \frac{\beta(\tau) S(\tau) I(\tau)}{S(\tau)+\alpha I(\tau)} e^{(d+\sigma) \tau} d \tau\right) \\
& >0, \quad \forall t>0 .
\end{aligned}
$$

In the case where $E_{0}>0, I_{0}=0$, then $E(t)>0$, for any $t>0, \dot{I}(0)=k E_{0}>0$. Therefore, $(S(t), E(t), I(t), Q(t), R(t)) \notin$ $\partial \Gamma_{0}$ for sufficiently small $t>0$. That is to say, for any $\left(S_{0}, E_{0}, I_{0}, Q_{0}, R_{0}\right) \notin\{(S, 0,0, Q, R): S \geq 0, Q \geq 0, R \geq$ $0\},\left(S_{0}, E_{0}, I_{0}, Q_{0}, R_{0}\right) \notin M_{\partial}$. This implies that $M_{\partial} \subseteq$ $\{(S, 0,0, Q, R): S \geq 0, Q \geq 0, R \geq 0\}$ and $P_{0}$ is the only fixed point of $P$ and acyclic in $\partial \Gamma_{0}$. Moreover, Lemma 6 implies that $P_{0}$ is an isolated invariant set in $\Gamma$ and $W^{s}\left(P_{0}\right) \cap \Gamma_{0}=\varnothing$, where $W^{s}\left(P_{0}\right)$ is the stable set of $P_{0}$. By the acyclicity theorem on uniform persistence for maps [34], it follows that $P$ is uniformly persistent with respect to $\left(\Gamma_{0}, \partial \Gamma_{0}\right)$.

Furthermore, taking advantage of Theorem 1.3.6 in Zhao [34], $P$ has a fixed point. Consider

$$
\left(\left(S^{*}(0), E^{*}(0), I^{*}(0), Q^{*}(0), R^{*}(0)\right)\right) \in \Gamma_{0} .
$$

Then we see that $S^{*}(0) \geq 0, E^{*}(0)>0, I^{*}(0)>0, Q^{*}(0) \geq$ 0 , and $R^{*}(0) \geq 0$. We further prove that $S^{*}(0)>0, Q^{*}(0)>$ 0 , and $R^{*}(0)>0$. Suppose not, if $S^{*}(0)=0$, from the first equation of model (1), we derive that

$$
\frac{d S^{*}(t)}{d t} \geq \Lambda-\left(d+\beta(t) \alpha^{-1}\right) S^{*}(t)+\eta R^{*}(t),
$$

with $S^{*}(0)=S^{*}(n \omega)=0, n=1,2,3 \ldots$ Then we have

$$
\begin{aligned}
S^{*}(n \omega) \geq & e^{-\int_{0}^{n \omega}\left(d+\beta(s) \alpha^{-1}\right) d s} \\
& \times\left(S^{*}(0)+\int_{0}^{n \omega} e^{\int_{0}^{\tau}\left(d+\beta(s) \alpha^{-1}\right) d s}\left(\Lambda+\eta R^{*}(\tau)\right) d \tau\right)
\end{aligned}
$$$$
>0 \text {. }
$$

The periodicity of $S^{*}(t)$ implies $S *(t)>0$, for all $t>0$. Similarly, $Q^{*}(0)>0$ and $R^{*}(0)>0$. Thus, $u\left(t,\left(S^{*}(0), E^{*}(0), I^{*}(0), Q^{*}(0), R^{*}(0)\right)\right) \in \operatorname{Int} \mathbb{R}_{+}^{5}$ and $\left(S^{*}(t)\right.$, $\left.E^{*}(t), I^{*}(t), Q^{*}(t), R^{*}(t)\right)$ is a positive $\omega$-periodic solution of model (1). This completes the proof.

Remark 8. Theorem 7 tells us that when the basic reproduction number $R_{0}>1$, model (1) admits at least one positive periodic solution, and the infectives are ultimately bounded below by some positive constant $\varepsilon$, which indicates that HFMD becomes an endemic disease.

Remark 9. From Theorems 4 and 7, we can conclude that $R_{0}$ is the threshold parameter for the extinction and the uniform persistence of the disease, which partially answer our proposed question in the introduction: "what is the disease dynamics of the HFMD in Wenzhou?”.

\section{Simulation Results via Statistical Data in Wenzhou}

In this section, by using model (1), we simulate the reported data of HFMD in Wenzhou and carry out the sensitivity analysis based on the parameters.

Thanks to the insightful work of Liu [17], Ma et al. [20], and Yang et al. [19], we estimate the values of all parameters of model (1) in details:

(i) the recruitment rate of susceptible $\Lambda$ : from the statistical data of Wenzhou population [24], one can know that the average birth number of Wenzhou population from 2005 to 2010 is 102729; then we adopt the weekly birth population number $\Lambda=1975$;

(ii) the natural mortality rate $d$ : in this paper, we take the average death rate of Wenzhou population as the natural mortality rate $d \approx 0.642 \%$ [24];

(iii) the transmission rate $\beta(t)$ : there are some epidemiological models using sinusoidal function $\left(\beta(t)=\beta_{0}+\right.$ $\left.\beta_{1} \sin (\omega t+\phi)\right)$ for the seasonal varying transmission rate $[16,17,19,20]$; in this paper, we use the periodic function $\beta(t)=\beta_{0}+\beta_{1} \sin (\omega t+\phi)$ with period $\omega$ (here $\omega=52$ weeks) as the transmission rates; by using the least-square fitting of the numbers of HFMD $I(t)$, we can estimate the values of $\beta_{0}, \beta_{1}$, and $\phi$;

(iv) the "psychological" effect $\alpha$ : in this paper, we adopt $\alpha=0.5$; it would be likely to note that when $\alpha=1$, the transmission rate is the classical standard incidence rate;

(v) the mean incubation period $1 / \sigma$ : as discussed in the introduction section, a person who is exposed to HFMD viruses will exhibit the symptoms after 3 to 7 days, for simplicity; in this paper, we assume that the average incubating time is 4 days, then the mean incubation period $1 / \sigma$ can be determined by $4 / 7$;

(vi) the quarantined rate $k$ : some of the infected people will be hospitalized to isolate for treatment; the ratio of these quarantined in infectious is called the quarantined rate; based on the statistical data, we can determine $k=0.007177$;

(vii) the infected recover rate $\gamma_{1}$ : we assume that the average recovery time of infectious is 8 days; then the recovery rate is $7 / 8=0.875$;

(viii) the quarantined recover rate $\gamma_{2}$ : we assume that the average recovery time of quarantined is 16 days; then the recovery rate is $7 / 16=0.4375$;

(ix) the disease-induced mortality $\delta_{1}$ and $\delta_{2}$ : we assume that the death comes from the quarantined; that is, $\delta_{1}=0$; and by using the least-square fitting of HFMD number $I(t)$, we can determine $\delta_{2}=0.024887$;

(x) the progression rate $\eta$ : by using the least-square fitting of HFMD number $I(t)$, we can determine $\eta=0.015$.

And the number of the initial susceptible population at the end of 2010, $S(0)=9,122,100$, is obtained from 
the Wenzhou City Population and Family Planning Commission [24]. The numbers of the initial infected $I(0)$ and quarantined $Q(0)$ can be obtained from the reported data of HFMD. Because the numbers of the initial exposed population $E(0)$ and the recovered population $R(0)$ cannot be obtained directly, we derive $E(0)$ by $k$ and $R(0)$ by $\gamma_{1}$ and $k \gamma_{2}$.

The numerical simulation results of the model (1) about the number of HFMD infectious are shown in Figures 2-4.

In Figure 1, we show the spread dynamics of HFMD in Wenzhou from March 2010 to November 2013, and the parameters in model (1) undefined in the above are taken as

$$
\begin{gathered}
I(0)=368, \quad E(0)=210, \\
Q(0)=322, \quad R(0)=323, \\
\beta(t)=0.91+0.39 \sin \left(\frac{\pi t}{26}+1.75\right) .
\end{gathered}
$$

In this case, $R_{0} \approx 0.9833<1$; the disease-free equilibrium $P_{0}=(307792,0,0,0,0)$ of model (1) is globally asymptotically stable, which means the HFMD disease will vanish after long time. The HFMD will die out, but is it always true?

For the sake of learning the spread dynamics of HFMD in Wenzhou further, we display the simulation results via the statistical data 2011-2012 (Figure 2) and 2012-2013 (Figure 4), respectively. In Figure 2, with parameters

$$
\begin{gathered}
I(0)=136, \quad E(0)=78, \\
Q(0)=119, \quad R(0)=167, \\
\beta(t)=0.9335+0.5225 \sin \left(\frac{\pi t}{26}+1.575\right) ;
\end{gathered}
$$

then $R_{0} \approx 1.0259>1$, which means that HFMD becomes an endemic disease in Wenzhou. In this case, from Theorems 4 and 7, one can know that model (1) has at least one positive periodic solution which is uniformly persistent. Figures 3(a) and $3(\mathrm{~b})$, with the same parameters as in Figure 2, confirm this conclusion.

In Figure 4, with parameters

$$
\begin{gathered}
I(0)=234, \quad E(0)=134, \\
Q(0)=205, \quad R(0)=206, \\
\beta(t)=0.908+0.47 \sin \left(\frac{\pi t}{26}+1.575\right),
\end{gathered}
$$

and, hence, $R_{0} \approx 0.9723<1$, which means the HFMD disease will vanish after long time.

Comparing Figure 1 with Figures 2 and 4, one can see that with partial or the whole HFMD data in Wenzhou from 2010 to 2013, with different time periodic data, then by using least-square fitting method, we can obtain different periodic transmission rate $\beta(t)$, and, hence, obtain different $R_{0}$, so we can obtain different disease spread dynamics and draw different conclusions. This difference is induced by different data and initial values.

For the sake of learning the effects of the quarantined rate $(k)$ and infected recovery rate $\left(\gamma_{1}\right)$ on the controlling

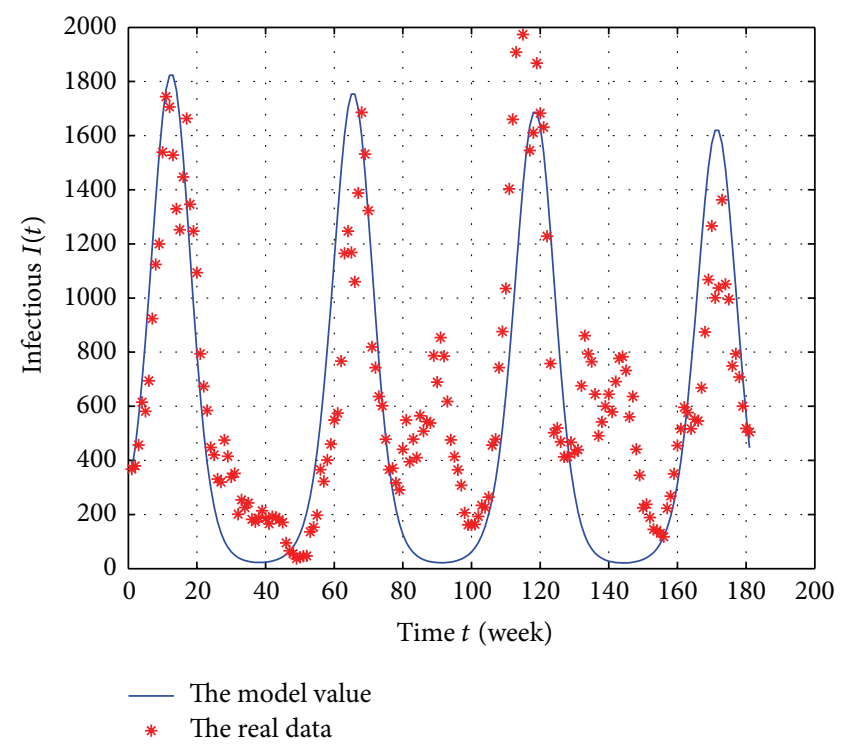

Figure 1: The solid curve represents the simulation results and the stars are the weekly data from 2010 to 2013 reported by the Wenzhou CDC.

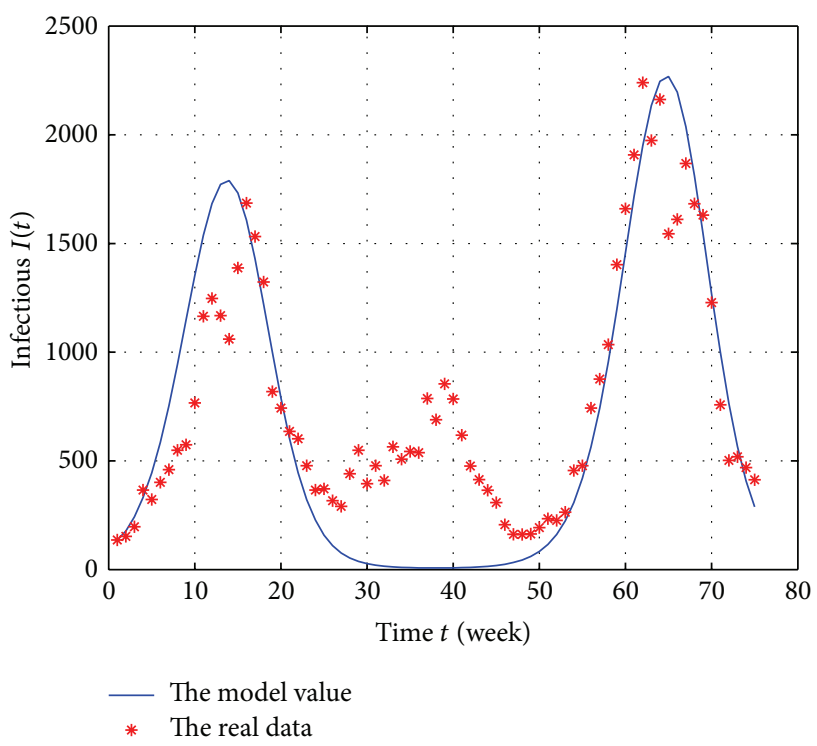

FIGURE 2: The solid curve represents the simulation results and the stars are the weekly data from 2011 to 2012 reported by the Wenzhou CDC.

of disease spreading, we consider the relations between the reproduction number $R_{0}$ with $k$ and $\gamma_{1}$, respectively. Figure 5(a) shows that the larger quarantined rate $k$ is, the less $R_{0}$ is; and that is to say, quarantine has a positive impact on controlling the spread of disease. Figure 5(b) has the similar tendency with Figure 5(a); that is, the larger quarantined rate $\gamma_{1}$ is, the less $R_{0}$ is. And $\gamma_{1}=7 / T$ ( $T$ is the cycle of treatment); hence, we can conclude that the less the cycle of treatment $T$ is, the less $R_{0}$ is. 


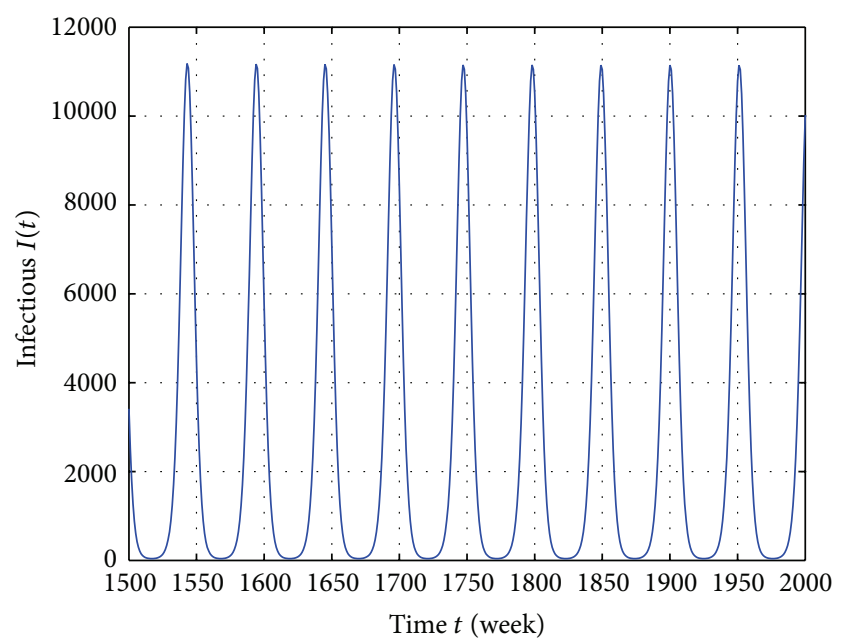

(a)

FIGURE 3: The population dynamics of model (1) with parameters

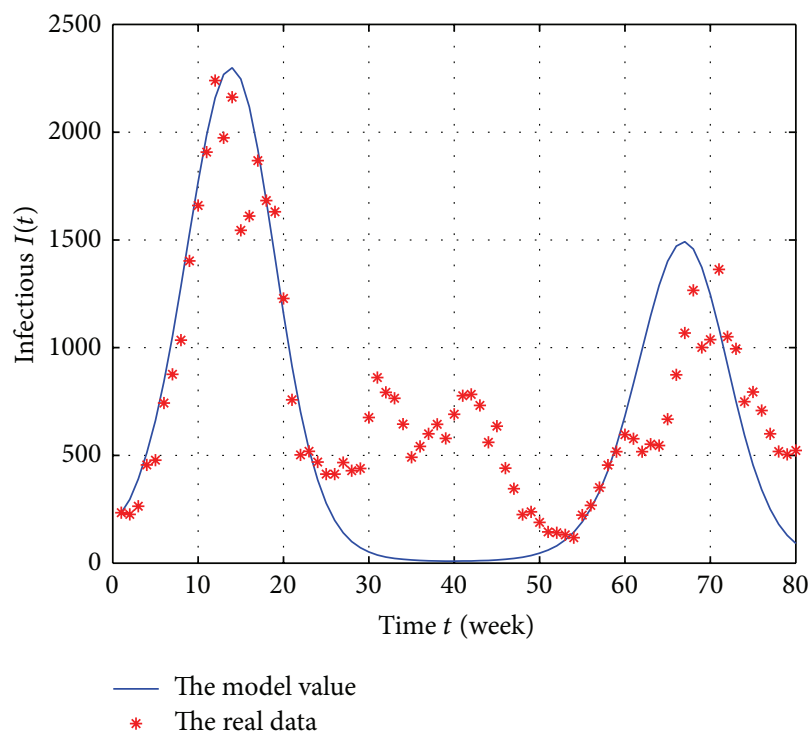

FIGURE 4: The solid curve represents the simulation results and the stars are the weekly data from 2012 to 2013 reported by the Wenzhou CDC.

\section{Conclusions and Discussions}

In this paper, we construct an SEIQRS epidemic model with periodic transmission rates to investigate the spread of seasonal HFMD in Wenzhou. We show that the global dynamics of the HFMD model (1) can be governed by its reproduction number $R_{0}$ : if $R_{0}<1$; the disease-free equilibrium $P_{0}=(\Lambda / d, 0,0,0,0)$ of model (1) is globally asymptotically stable, which means the disease will die out; while $R_{0}>1$, model (1) has at least one positive periodic solution and is uniformly persistent, which indicates that HFMD becomes an endemic disease; that is, the HFMD will break out.

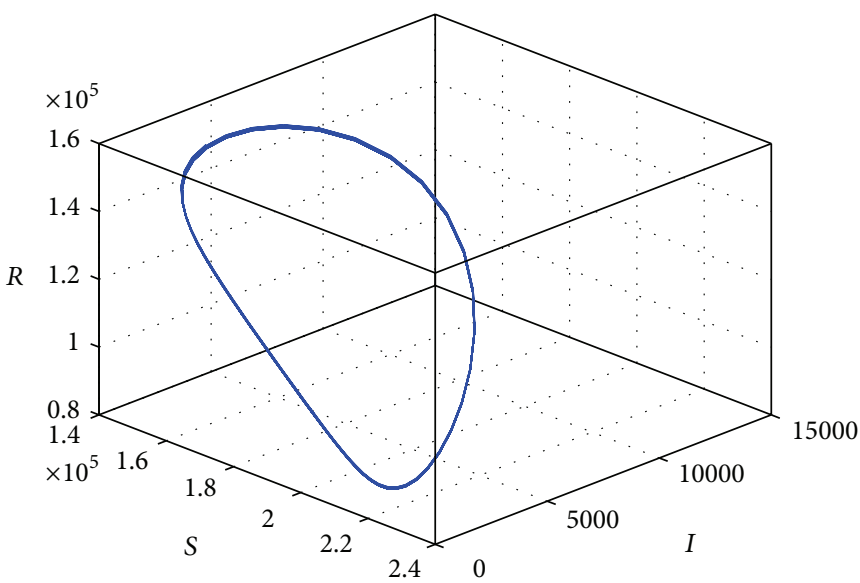

(b)

(43). (a) Time series plots; (b) phase portraits (periodic solution).

Epidemiologically, we partially provide answers to the two questions proposed in the introduction: what is the spread dynamics of the HFMD in Wenzhou? How can we control the HFMD spread in Wenzhou?

From Figures 1, 2, and 4, we can obtain different disease spread dynamics and draw different conclusions based on the different data and initial values. In fact, a mathematical model may help to explain a system, to study the effects of different components, and to make predictions about disease spreading behaviour. We need to point out that all these three figures are partly explaining and predicting the spread dynamics of the HFMD in Wenzhou. Besides, in the cases of Figures 1, 2, and 4, we obtain different basic reproduction numbers $R_{0}$, which can mathematically help us to determine whether an infectious disease can spread or not. There are two values of $R_{0}$ less than 1 , and close to 1 . It is worthy to note that, in the HFMD model (1), we assume that the whole population of Wenzhou (more than 9 million people) is homogeneously mixing. If we try to take children under five-17 years old and their family as the susceptible population (about 1 million people), the value of $R_{0}$ would be larger. All in all, we can conclude that the HFMD in Wenzhou persists under current circumstances; that is, the HFMD becomes an endemic disease and will break out in Wenzhou.

From Figure 5, we find that the quarantined rate $(k)$ and the infected recovery rate $\left(\gamma_{1}\right)$ are very beneficial to control the disease outbreak. That is to say, for controlling the HFMD spread in Wenzhou, we must increase the quarantined rate or decrease the treatment cycle.

In [20], based on the statistical data HFMD in Shandong province, China, Ma et al. indicated that the quarantined rate $k$ has little effect on controlling the disease spread. This is very different from our present results. It is reported that the HFMD incidence strongly depends on the climate [19]. We think this difference may be induced by different climate: Shandong province is in north China, and Wenzhou is in south China. 


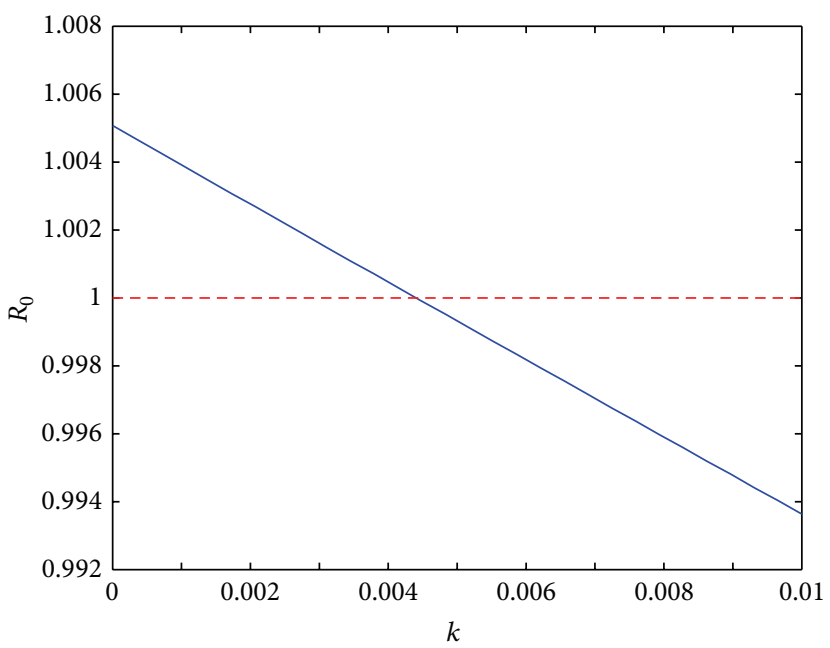

(a)

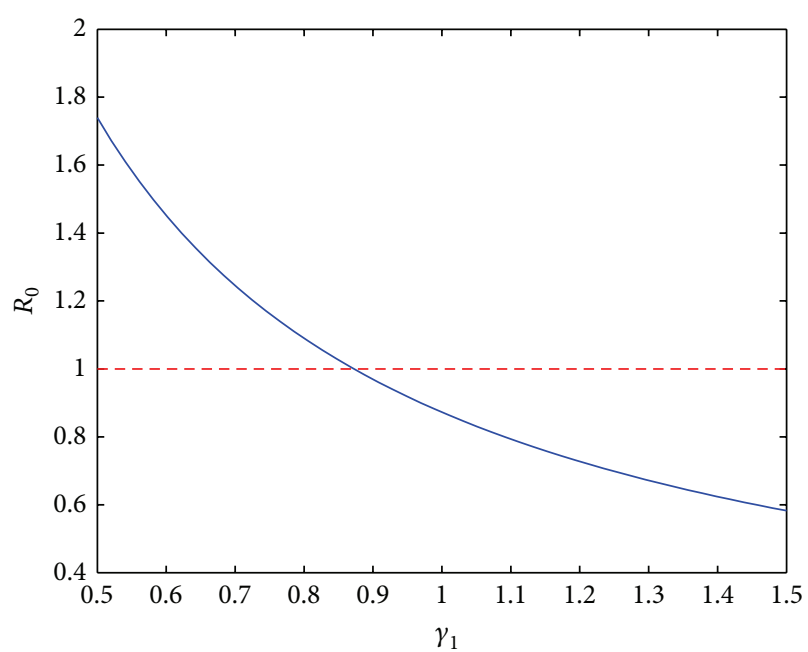

(b)

FIGURE 5: The influence of the quarantined rate $k$ (a) and infected recover rate $\gamma_{1}$ (b) on $R_{0}$.

Furthermore, from Figures 1, 2, and 4, one can see that the fittings have some flaws because of oscillation peak in the data of summer 2011 and 2012. We think this phenomenon may represent an alarm for next year prevalence. In addition, there may be more suitable incidence rate $\beta(t)$ to fit the spread dynamics of the HFMD in Wenzhou. This is desirable in future studies.

It is well known that no vaccine currently exists to protect individuals from infection with the viruses that cause HFMD, but such vaccines are being developed [35]. HFMD is highly contagious and is transmitted by oropharyngeal secretions such as saliva or nasal mucus, by direct contact or fecal-oral transmission. Preventive measures include avoiding direct contact with infected individuals (including keeping infected children home from school), proper cleaning of shared utensils, disinfecting contaminated surfaces, and proper hand hygiene. These measures have been proved to be effective in decreasing the transmission of the viruses responsible for HFMD $[1,35]$.

\section{Conflict of Interests}

The authors declare that there is no conflict of interests regarding the publication of this paper.

\section{Acknowledgments}

The authors would like to thank Jing-An Cui and Meng Ye (Beijing University of Civil Engineering and Architecture) and Maoxing Liu and Yangjun Ma (North University of China) for very helpful suggestions and comments which led to the improvement of the original paper. This research is supported by the National Science Foundation of China (61373005) and Zhejiang Provincial Natural Science Foundation (LY12A01014).

\section{References}

[1] Centers for Disease Control and Prevention, "About hand, foot, and mouth disease," http://www.cdc.gov/hand-foot-mouth.

[2] J. P. Lott, K. Liu, M. Landry et al., "Atypical hand-foot-andmouth disease associated with Coxsackievirus A6 infection," Journal of the American Academy of Dermatology, vol. 69, no. 5, pp. 736-741, 2013.

[3] L.-X. Mao, B. Wu, W.-X. Bao et al., "Epidemiology of hand, foot, and mouth disease and genotype characterization of Enterovirus 71 in Jiangsu, China," Journal of Clinical Virology, vol. 49, no. 2, pp. 100-104, 2010.

[4] M. Y. Liu, W. Liu, J. Luo et al., "Characterization of an outbreak of hand, foot, and mouth disease in nanchang, China in 2010," PLoS ONE, vol. 6, no. 9, Article ID e25287, 2011.

[5] Q. Zhu, Y. Hao, J. Ma, S. Yu, and Y. Wang, "Surveillance of hand, foot, and mouth disease in Mainland China (2008-2009)," Biomedical and Environmental Sciences, vol. 24, no. 4, pp. 349356, 2011.

[6] M. Zeng, Y. F. Li, X. H. Wang et al., "Epidemiology of hand, foot, and mouth disease in children in Shanghai 2007-2010," Epidemiology and Infection, vol. 140, no. 6, pp. 1122-1130, 2012.

[7] L. W. Ang, B. K. Koh, K. P. Chan, L. T. Chua, L. James, and K. T. Goh, "Epidemiology and control of hand, foot and mouth disease in Singapore, 2001-2007," Annals of the Academy of Medicine Singapore, vol. 38, no. 2, pp. 106-112, 2009.

[8] S. Blomqvist, P. Klemola, S. Kaijalainen et al., "Co-circulation of coxsackieviruses A6 and A10 in hand, foot and mouth disease outbreak in Finland," Journal of Clinical Virology, vol. 48, no. 1, pp. 49-54, 2010.

[9] Y. L. Hii, J. Rocklöv, and N. Ng, "Short term effects of weather on Hand, foot and mouth disease," PLoS ONE, vol. 6, no. 2, Article ID e16796, 2011

[10] S. S. Y. Wong, C. C. Y. Yip, S. K. P. Lau, and K. Y. Yuen, "Human enterovirus 71 and hand, foot and mouth disease," Epidemiology and Infection, vol. 138, no. 8, pp. 1071-1089, 2010.

[11] J.-F. Wang, Y.-S. Guo, G. Christakos et al., "Hand, foot and mouth disease: spatiotemporal transmission and climate," International Journal of Health Geographics, vol. 10, article 25, 2011. 
[12] N. Roy and N. Haider, "Compartmental modeling of hand, foot and mouth infectious disease (HFMD)," Research Journal of Applied Sciences, vol. 5, no. 3, pp. 177-182, 2010.

[13] N. Roy, "Mathematical modeling of hand-foot-mouth disease: quarantine as a control measure," International Journal of Advanced Scientific Engineering and Technological Research, vol. 1, no. 2, pp. 34-44, 2012.

[14] E. Ma, T. Lam, K. C. Chan, C. Wong, and S. K. Chuang, "Changing epidemiology of hand, foot, and mouth disease in Hong Kong, 2001-2009," Japanese Journal of Infectious Diseases, vol. 63, no. 6, pp. 422-426, 2010.

[15] D. Zhu, X. Zhao, Y. Yao et al., "A new factor influencing pathogen detection by molecular assay in children with both mild and severe hand, foot, and mouth disease," Diagnostic Microbiology and Infectious Disease, vol. 76, no. 2, pp. 162-167, 2013.

[16] F. C. S. Tiing and J. Labadin, "A simple deterministic model for the spread of hand, foot and mouth disease (HFMD) in Sarawak," in Proceedings of the 2nd Asia International Conference on Modelling and Simulation (AMS '08), pp. 947-952, IEEE, May 2008.

[17] J. Liu, "Threshold dynamics for a HFMD epidemic model with periodic transmission rate," Nonlinear Dynamics, vol. 64, no. 12, pp. 89-95, 2011.

[18] X. Wang and Y. Xue, "Analysis of HFMD in China using a SEIQR model," Far East Journal of Mathematical Sciences, vol. 58, no. 1, pp. 83-98, 2011.

[19] J.-Y. Yang, Y. Chen, and F.-Q. Zhang, "Stability analysis and optimal control of a hand-foot-mouth disease (HFMD) model," Journal of Applied Mathematics and Computing, vol. 41, no. 1-2, pp. 99-117, 2013.

[20] Y. Ma, M. Liu, Q. Hou, and J. Zhao, "Modelling seasonal HFMD with the recessive infection in Shandong, China," Mathematical Biosciences and Engineering, vol. 10, no. 4, pp. 1159-1171, 2013.

[21] H. Ni, B. Yi, J. Yin et al., "Epidemiological and etiological characteristics of hand, foot, and mouth disease in Ningbo, China, 2008-2011," Journal of Clinical Virology, vol. 54, no. 4, pp. 342-348.

[22] J. Ma and Z. Ma, "Epidemic threshold conditions for seasonally forced SEIR models," Mathematical Biosciences and Engineering, vol. 3, no. 1, pp. 161-172, 2006.

[23] J. Dushoff, J. B. Plotkin, S. A. Levin, and D. J. D. Earn, "Dynamical resonance can account for seasonality of influenza epidemics," Proceedings of the National Academy of Sciences of the United States of America, vol. 101, no. 48, pp. 16915-16916, 2004.

[24] Wenzhou City Population and Family Planning Commission, "A survey of wenzhou population," http://www.wzrkjs.gov.cn, (in Chinese).

[25] Zhejiang Online Source for Disease Control and Prevention, "The statistic results of "Ten legal infectious diseases" in Zhejiang province in 2012," http://www.cdc.zj.cn, (in Chinese).

[26] D. Xiao and S. Ruan, "Global analysis of an epidemic model with nonmonotone incidence rate," Mathematical Biosciences, vol. 208, no. 2, pp. 419-429, 2007.

[27] G. Aronsson and R. B. Kellogg, "On a differential equation arising from compartmental analysis," Mathematical Biosciences, vol. 38, no. 1-2, pp. 113-122, 1978.

[28] M. W. Hirsch, "Systems of differential equations that are competitive or cooperative. II. Convergence almost everywhere," SIAM Journal on Mathematical Analysis, vol. 16, no. 3, pp. 423439, 1985.
[29] H. L. Smith and P. Waltman, The Theory of the Chemostat, vol. 13 of Cambridge Studies in Mathematical Biology, Cambridge University Press, Cambridge, UK, 1995.

[30] F. Zhang and X.-Q. Zhao, "A periodic epidemic model in a patchy environment," Journal of Mathematical Analysis and Applications, vol. 325, no. 1, pp. 496-516, 2007.

[31] N. Bacaër and S. Guernaoui, "The epidemic threshold of vector-borne diseases with seasonality. The case of cutaneous leishmaniasis in Chichaoua, Morocco," Journal of Mathematical Biology, vol. 53, no. 3, pp. 421-436, 2006.

[32] W. Wang and X.-Q. Zhao, "Threshold dynamics for compartmental epidemic models in periodic environments," Journal of Dynamics and Differential Equations, vol. 20, no. 3, pp. 699-717, 2008.

[33] L. Perko, Differential Equations and Dynamical Systems, Springer, New York, NY, USA, 2000.

[34] X.-Q. Zhao, Dynamical Systems in Population Biology, Springer, New York, NY, USA, 2003.

[35] N. Sarma, "Hand, foot, and mouth disease: current scenario and indian perspective," Indian Journal of Dermatology, Venereology, and Leprology, vol. 79, no. 2, pp. 165-175, 2013. 


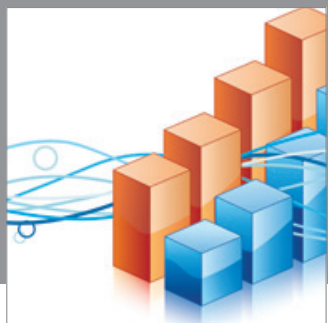

Advances in

Operations Research

mansans

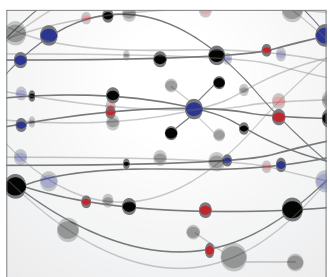

The Scientific World Journal
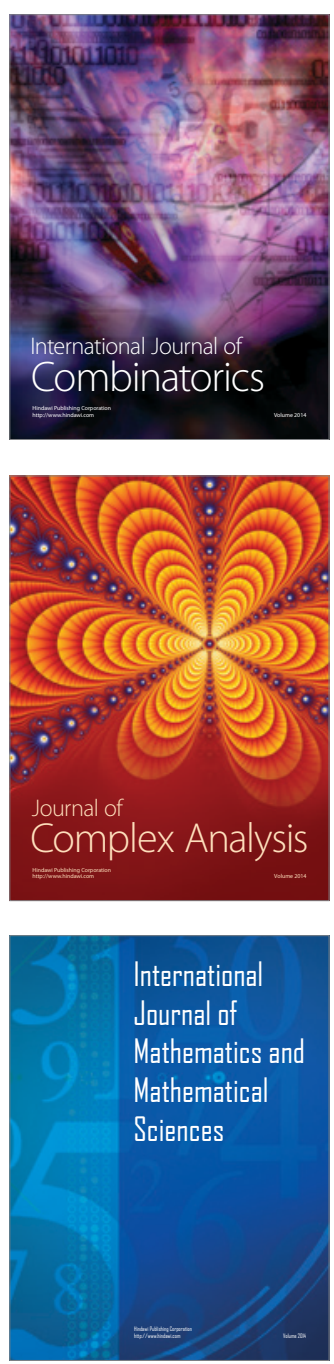
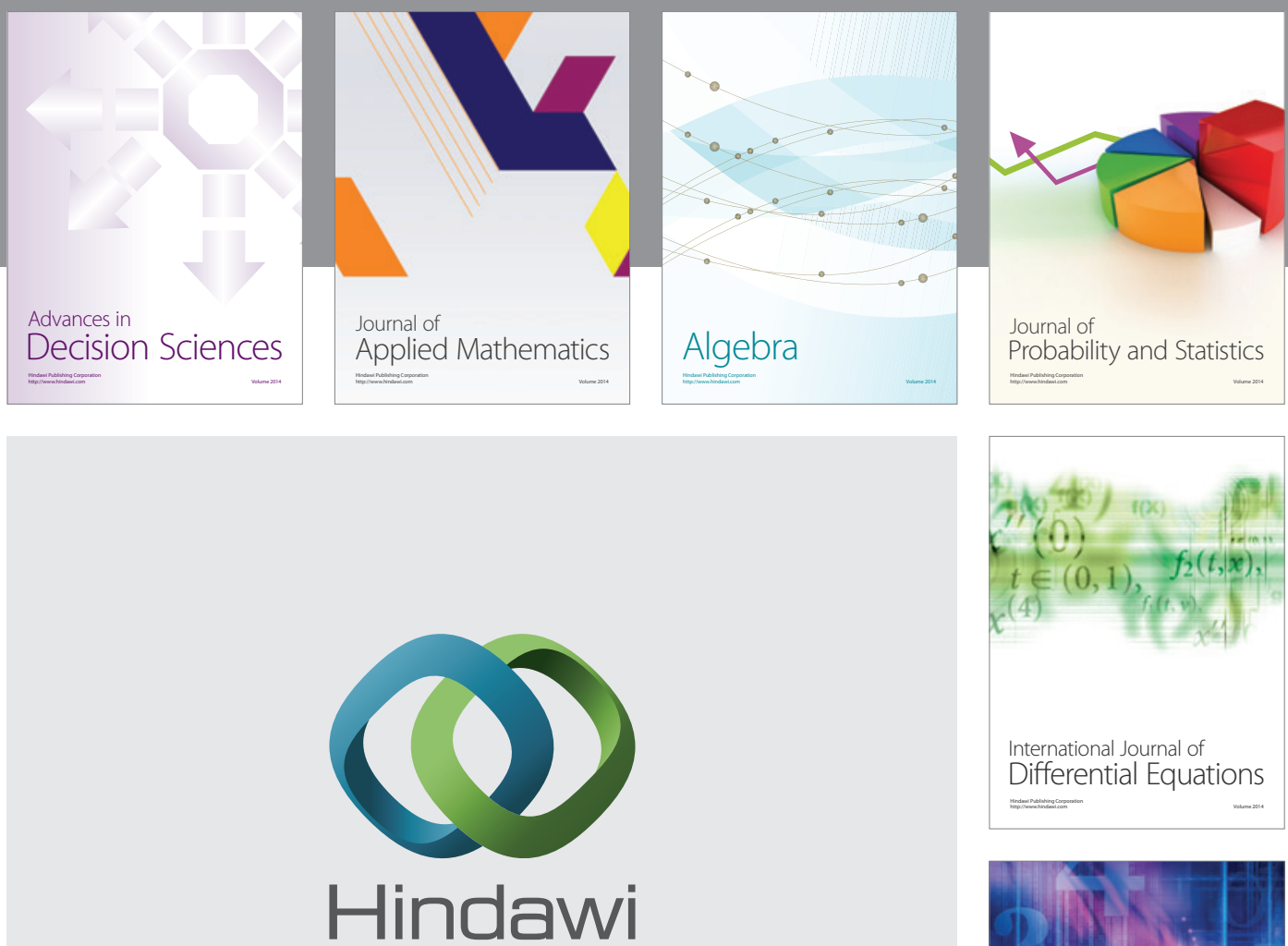

Submit your manuscripts at http://www.hindawi.com
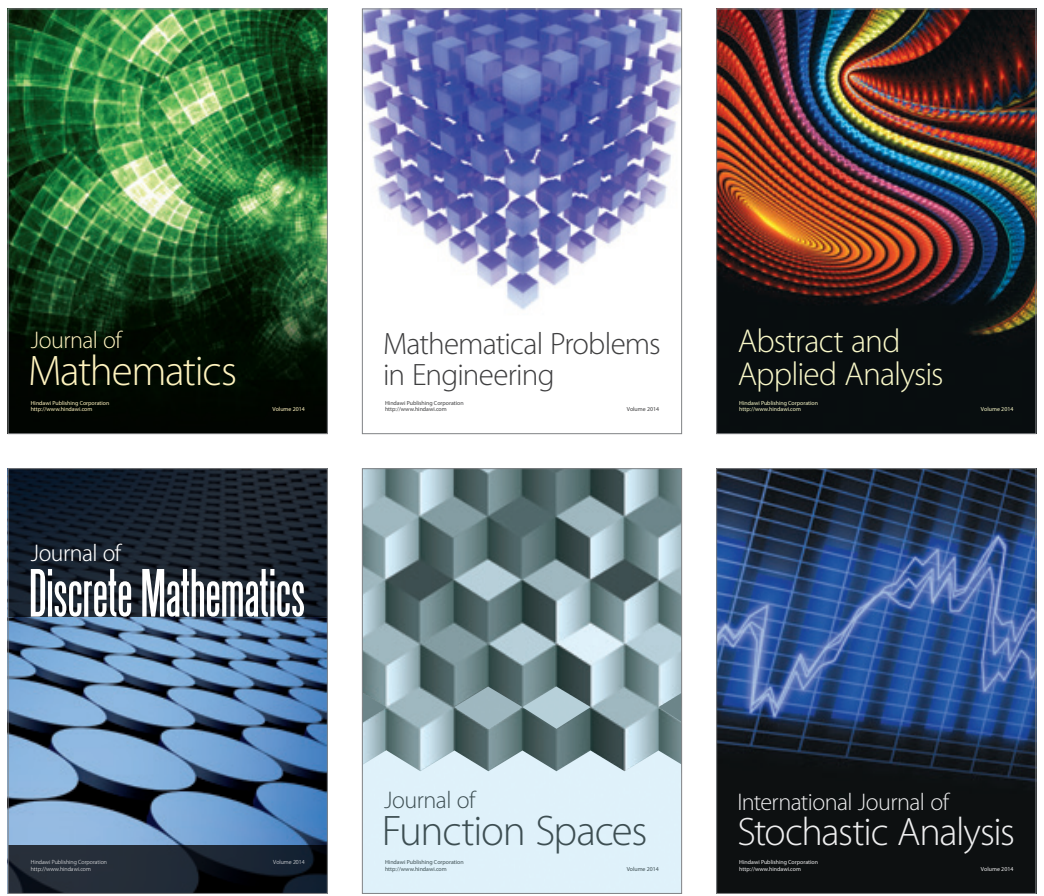

Journal of

Function Spaces

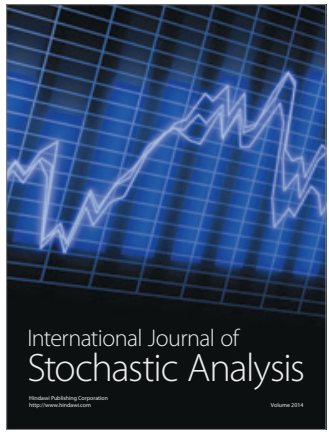

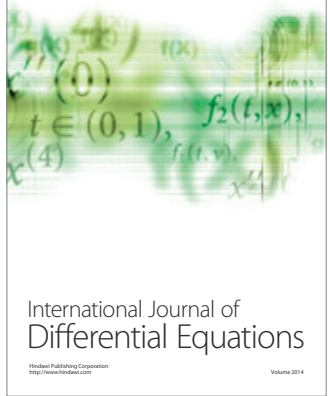
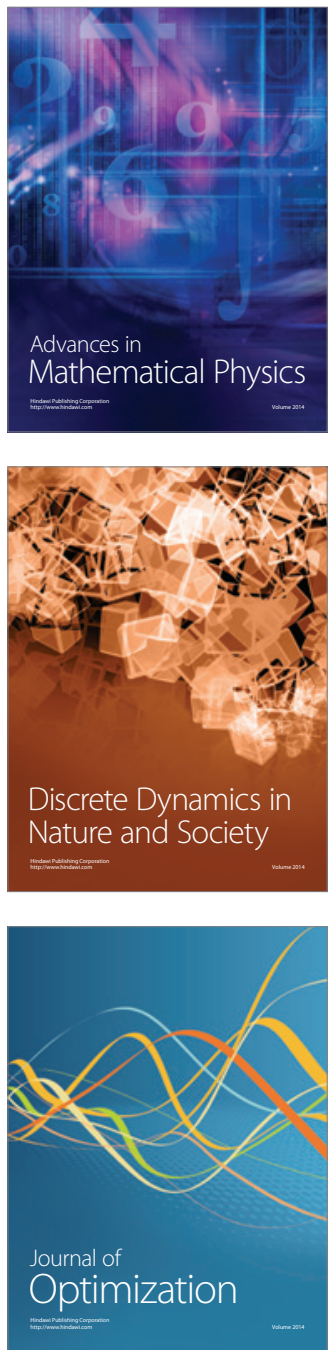